\title{
Oscillating motion of the Jovian Great Red Spot and Numerical Experiments with IG equation
}

\author{
Tadashi Asada ${ }^{1}$ and Isao Miyazaki² \\ ${ }^{1}$ Kyushu International University, Japan \\ ${ }^{2}$ Uruma City in Okinawa, Japan
}

(Received July 9, 2005; Revised February 27, 2006; Accepted March 4, 2006; Online published July 26, 2006)

\begin{abstract}
Position measurements of the Great Red Spot (GRS) on recent digital images of Jupiter were carried out to detect the longitudinal and latitudinal motion of the GRS. Its longitudinal oscillating motion may be interpreted as two types. One has a 57-day period and no latitudinal motion, the other has 86 days and probably has latitudinal motion. In 86 days, oscillation, the GRS seems to locate equatorward when it moves relatively westward, and poleward when it moves eastward. Numerical Experiments of the Intermediate Geostrophic (IG) equation show the same results for the motion of an eddy, and that a kind of wave collides with the eddy when it changes relative longitudinal motion from eastward to westward.
\end{abstract}

Key words: Jupiter, atmosphere, dynamics, GRS, oscillation, simulation.

\section{Introduction}

The longitudinal oscillating motion of the Great Red Spot (GRS) was found by a team from the New Mexico State University around 1970 . The motion is said to have a 90day period and a 0.8 degree amplitude in longitude. (Solberg, 1969; Reese, 1970, 1971, 1972; Reese and Beebe, 1976).

The mechanism of this oscillating motion is not known. Although it is said that some markings collide with the GRS every 88 days, no one has examined what kind of markings collide with the GRS and whether the GRS really shows such an oscillation if a collision occurs.

Trigo-Rodriguez et al. (2000) confirmed the 90-day oscillation of the GRS. They used a large number of images from 1993 to 1999 , and concluded that the period was $89.8 \pm 0.15$ days and the amplitude was $1.2 \pm 0.7^{\circ}$. But they did not pay attention to the importance of the latitudinal motion, nor give any mechanism for this oscillation.

Recent developments of imaging devices and image processing techniques allow us to get high quality images of Jupiter in digital format. We carried out position measurements of the GRS, detected the longitudinal oscillating motion, and examined the correlation of longitudinal and latitudinal ${ }^{1}$ motion.

Asada (1985a) speculated that an eddy oscillated in the latitudinal direction around the latitude of a peculiar westward zonal flow and that this latitudinal motion caused the longitudinal motion due to the zonal flows around the GRS. However, his simulation results with the IG equation was that the eddy collides with weak waves when it began to

Copyright (c) The Society of Geomagnetism and Earth, Planetary and Space Sciences (SGEPSS); The Seismological Society of Japan; The Volcanological Society of Japan; The Geodetic Society of Japan; The Japanese Society for Planetary Sciences; TERRAPUB

\footnotetext{
${ }^{1}$ In this paper, we use only planetographic latitude.
}

move westwards. This paper was written in Japanese and published as a contribution in the Journal of Yahata University, hence it seems to be valuable to show his results again.

\section{Imaging and Measurements}

Imaging of Jupiter were carried out at Uruma City, Okinawa, using a $40 \mathrm{~cm}$ Newtonian F6 reflector. Images were taken by web cam, ToUcam of Philips, which has $640 \times 480$ pixels of CCD, and saved as an AVI file of video image to PC. The image size on CCD is around 400 pixels when the apparent diameter of Jupiter is 40 arcsec. Hence, 1 pixel corresponds about 0.1 arcsec. With Registax, image-processing software, an AVI file is divided into many frames, and those frames are evaluated and ordered from best quality to worst. The better 430-460 frames were integrated and processed by the wavelet transformation. In Fig. 1, we show one such image, which is a green light component of a color image.

In order to measure the position of the Jovian markings, we needed to decide the acquisition time of each image as accurate as possible. Because the frame rate is $15 \mathrm{fps}$ (frames per second), it takes at least $30 \mathrm{sec}$ to record 450 frames. The central meridian longitude of Jupiter varies by 0.3 degree in $30 \mathrm{sec}$. In integration of many frames, we can choose one reference frame, and we adopt the time of this reference frame as the time of the integrated image. The accuracy of the time is within $1 \mathrm{sec}$. We got 87 images for the GRS from October 2003 to July 2004.

The amplitude of oscillating motion, 0.8 degree, corresponds to 0.3 arcsec at the disc center of the Jovian image, if the apparent diameter is 42 arcsec. The amplitude also corresponds to 3 pixels, if the Jovian image size on CCD is 420 pixels.

We used green light components of color images for measurement. The blue component has great contrast between 


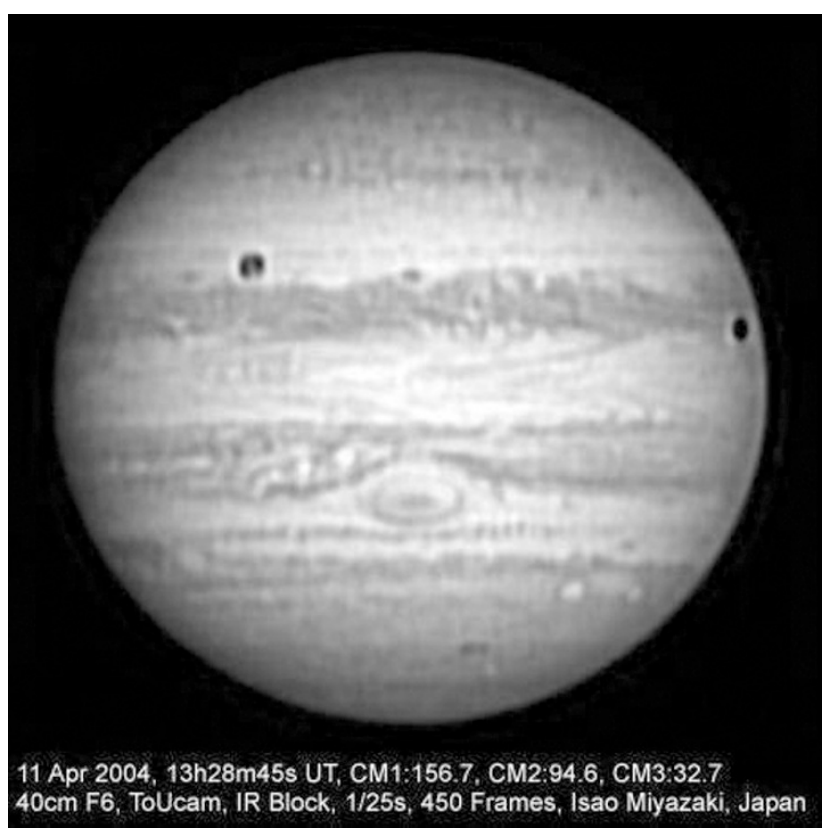

Fig. 1. Image of Jupiter from which we measured the longitude and latitude of the GRS. North is top.

the GRS and surroundings, but the limb or terminator is not well defined in blue. In the red component, the limb or terminator is well defined, but the GRS has almost the same brightness as the surroundings. Hence, only the green component can be used for our measurements.

We calculated the longitude and latitude from the position of the markings and of the limb and terminator. We made a program for this calculation using the IDL software for the image processing. For each image, the measurements of the markings' position were carried out for longitudes of the east (proceeding) end and west (following) end, and for latitudes of the north and south ends. Measurements of each end were made 10 times, and means and standard errors were derived. We calculated the eddy's central longitude as a mean of the longitude of the east and west end, and the error of longitude as a root mean square of the standard errors of the east and west ends. The central latitude is also calculated as a mean of the north and south latitudes, and the error of latitude as a root mean square of the standard errors for north and south ends.

In our previous measurements, we determined the position of the limb or terminator by just clicking the mouse. In this work, we improved the detection method of limb or terminator as follows:

- We decide on a threshold value for the limb or terminator as 5 or $12 \%$ of the maximum brightness of the Jovian disc, which depends on the image quality.

- We click a point of the limb or terminator with the mouse for east, west, north, and south ends of the image disc.

- Near the clicked point, the point of the threshold value of brightness was searched for. If the clicked point has less brightness than the threshold, the inner point is checked. If more brightness, the outer point is examined.
- At the point of the threshold of the brightness, the limb or terminator are extrapolated using the slope of brightness and threshold value.

This method was checked for its validity by measuring the same point some $10 \mathrm{~min}$ apart. In the previous method, the longitude decreased 2 or 3 degrees in 30 or $40 \mathrm{~min}$. This is because the limb or terminator was clicked inner than the actual and the diameter was estimated to be smaller. With this new method, such a decrease of longitude was not so obvious.

Moreover, we checked the image orientation from the position of the center of gravity for the right and left halves of the Jovian disc. If the $y$ value of the two centers of gravity is different by more than 1 pixel, we rotated the image clockwise or counterclockwise so that the north-south direction of the Jovian disc coincides with the $y$ direction of the frame.

\section{Results of Measurements}

Longitude variation of the GRS is shown in Fig. 2. Here the longitude is in System III. The errors are too small to see in the figure.

First, we fit the linear function of time to the longitude variation of the GRS, but the residuals were not small at the beginning of our analysis period. Hence, we made the least-squares approximation with the quadratic of time as follows, and derived the residuals.

$$
\text { longitude }=0.644 \times 10^{-4} t^{2}+0.273 t-20.942,
$$

where $t$ is day from 10 October 2003 21:19:49 UT. The coefficient of quadratic term was positive, and this means that the GRS had positive acceleration. For these residuals, we made a formal Fourier transformation as follows:

$$
\begin{aligned}
& a_{k}=\frac{1}{N_{o}} \Sigma_{i} x_{i} \cos \left(\frac{2 \pi t_{i}}{p_{k}}\right), \\
& b_{k}=\frac{1}{N_{o}} \Sigma_{i} x_{i} \sin \left(\frac{2 \pi t_{i}}{p_{k}}\right), \quad c_{k}=\sqrt{a_{k}^{2}+b_{k}^{2}},
\end{aligned}
$$

GRS Drift Chart

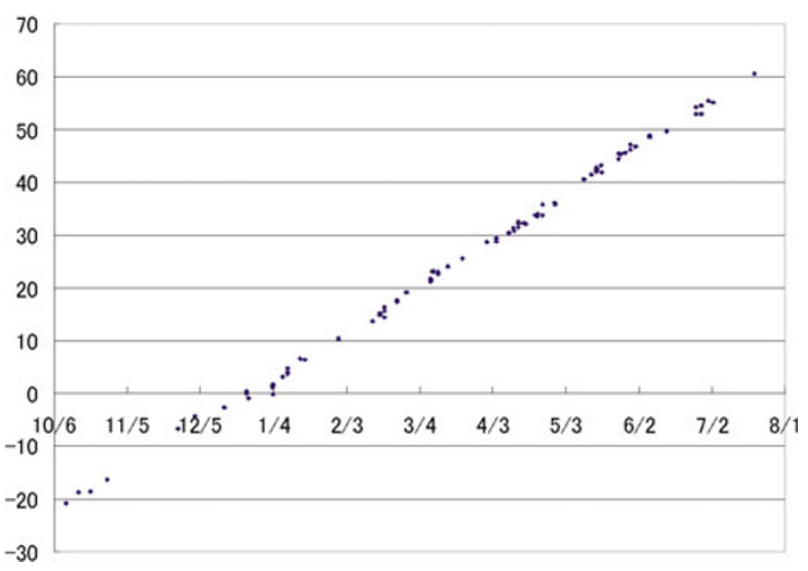

Fig. 2. Longitude variation of the GRS. Longitude is in System III. Before 3 January, the longitude is subtracted $360^{\circ}$ from the real value, in order for these values to be continuous to the longitudes after 3 January. 
Fourier components for the logitudinal motion of GRS

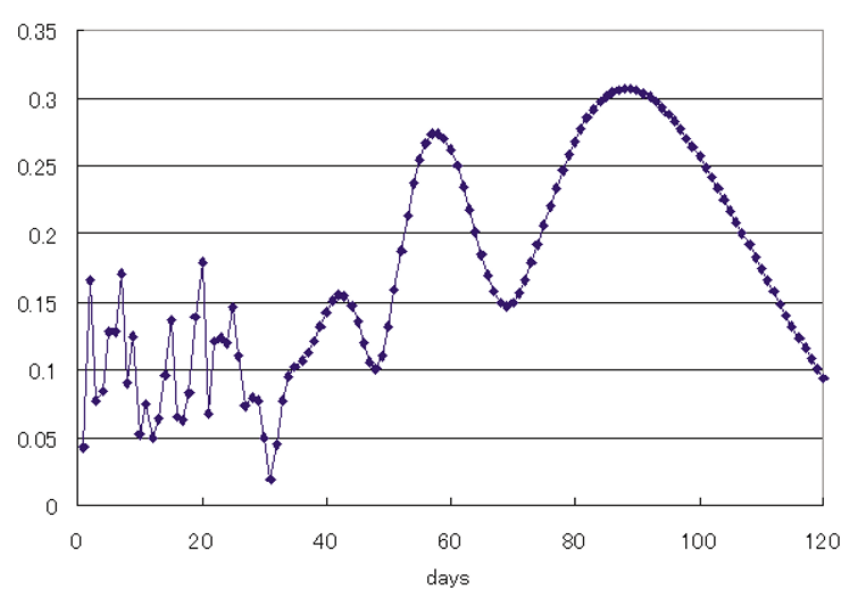

Fig. 3. Fourier component in the motion of the GRS. The unit of vertical axis is degree.

Table 1. The correlation coefficients of 55-60 and 85-90 days periods for the longitude and latitude motions of the GRS.

\begin{tabular}{ccc}
\hline $\begin{array}{c}\text { Period } \\
\text { (days) }\end{array}$ & Longitude & Latitude \\
\hline 55 & 0.5501 & 0.2122 \\
56 & 0.5777 & 0.1913 \\
57 & 0.5906 & 0.1724 \\
58 & 0.5901 & 0.1572 \\
59 & 0.5781 & 0.1461 \\
60 & 0.5565 & 0.1389 \\
\hline 85 & 0.5595 & 0.3106 \\
86 & 0.5608 & 0.3206 \\
87 & 0.5602 & 0.3297 \\
88 & 0.5587 & 0.3378 \\
89 & 0.5563 & 0.3449 \\
90 & 0.5529 & 0.3512 \\
91 & 0.5488 & 0.3568 \\
92 & 0.5439 & 0.3619 \\
93 & 0.5382 & 0.3668 \\
94 & 0.5317 & 0.3716 \\
95 & 0.5243 & 0.3765 \\
\hline
\end{tabular}

where $N_{o}, x_{i}, t_{i}$ and $p_{k}$ are the total number of data, the residual, time in days, and period which we set from 1 to 120 days. Although the time intervals of data $\left(t_{i}-t_{i-1}\right)$ are not constant, we think this result suggests the relative scale for the oscillation components. The results of this transformation are shown in Fig. 3.

From Fig. 3, it can be seen that there are two peaks. One is of a 58-day period, and the other is 88 days. Around these two components (55-60 and 85-95 days), we made the least-square fit of oscillation to the latitudinal motion and to the residuals of the longitudinal motion. The correlation coefficients are summarized in Table 1. From this table,
Oscillation of GFS
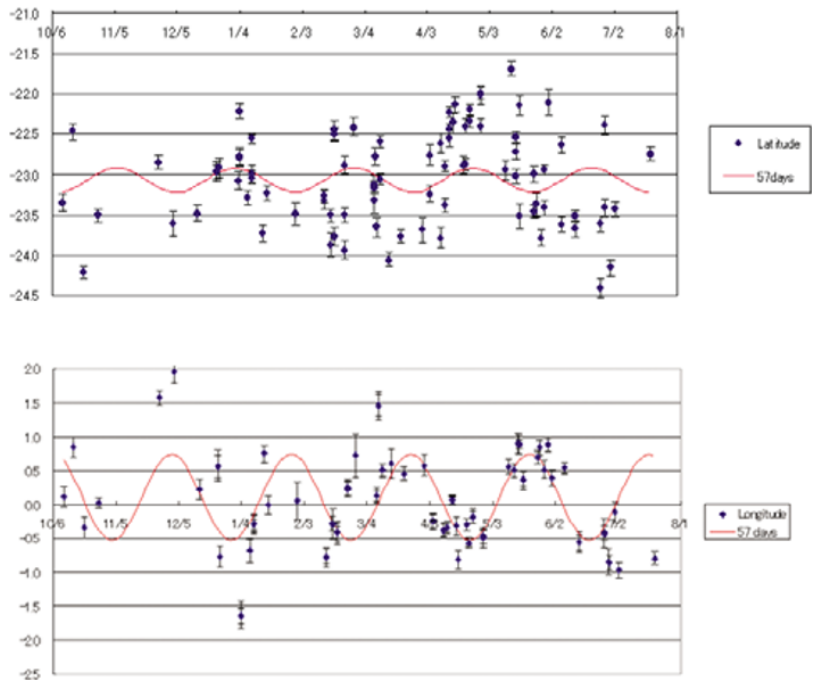

Fig. 4. The position variation of the GRS. Top is latitude, bottom is residuals in longitude. Error bars are the root-mean-square of standard error for north and south ends (latitude), and for east and west ends (longitude). The least-square fit of 57 days period components are drawn as lines.

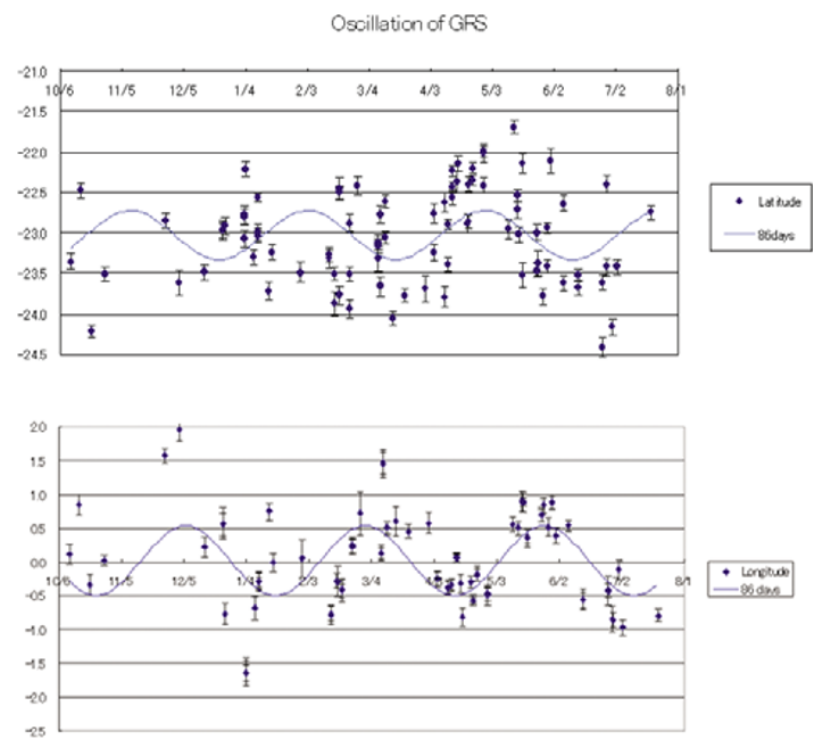

Fig. 5. The same as the previous figure, but for the 86-day period.

the coefficients of the longitudinal motion are almost the same for both 55-60 and 85-95 days, but the coefficients of the latitudinal motion for 85-95 days are almost twice those of 55-60 days. The maximum value of the correlation coefficients of longitude is 0.5906 for 57 days and 0.5605 for 86 days.

The results of fitting with these two periods are shown in Figs. 4 and 5. From Fig. 4 we can see the amplitude of longitudinal motion is about 0.63 degree for the 57 -day period, and from Fig. 5, the amplitude is 0.51 degree for 86 days. These amplitudes are small compared to the values obtained by New Mexico State University. The amplitudes of latitudinal motion are 0.15 degree for the 57 days component from Fig. 4, and 0.30 degree for 86 days from Fig. 5. These values are well below the resolution of our telescope, but 


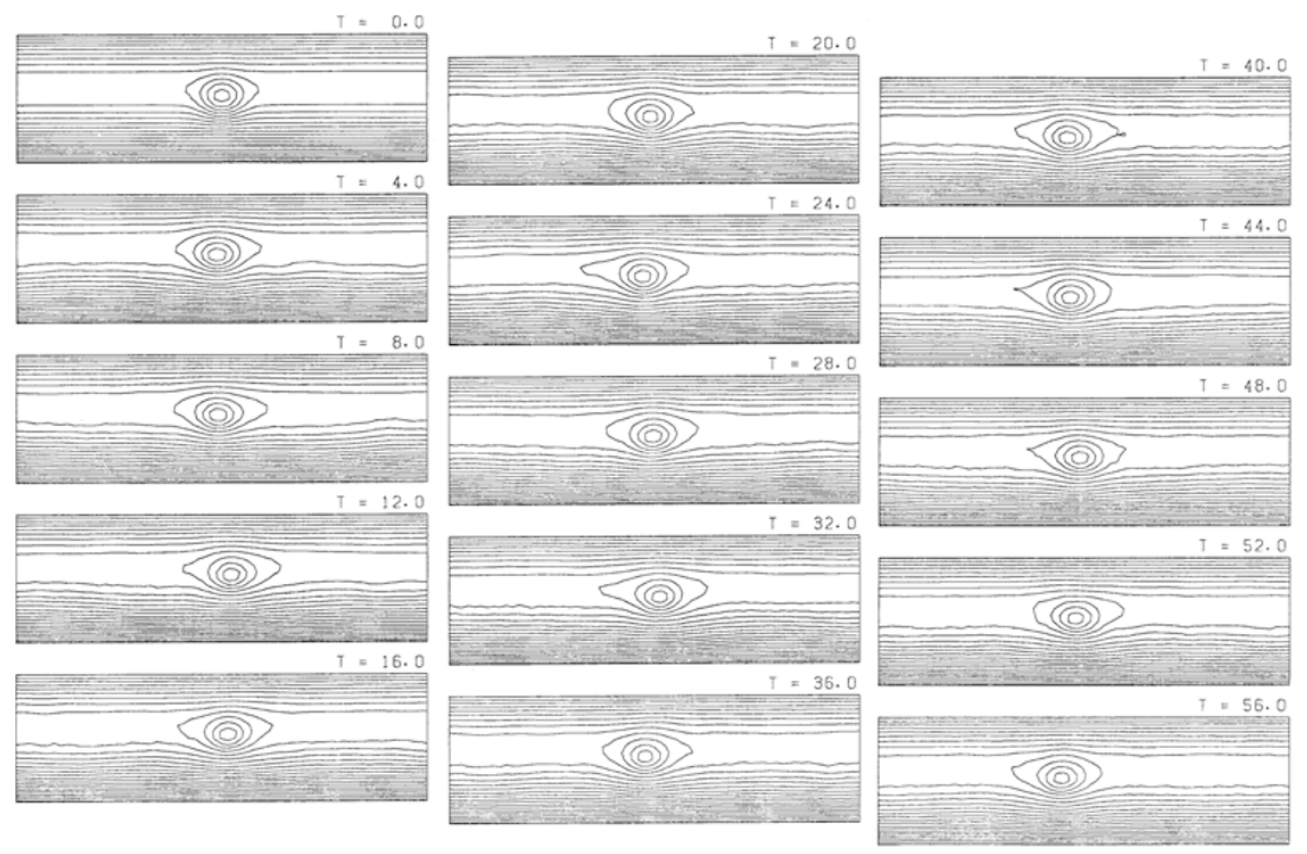

Fig. 6. The contour map of normalized surface elevation $\eta$ of our numerical experiments results. Each box shows our calculation region.

the peak-to-peak amplitude of 86 days is almost the limit of the resolution. The correlation coefficients are 0.1724 for 57 days, and 0.3206 for 86 days.

In conclusion, we may interpret the longitudinal oscillating motion as of two types.

- Type I: This component has a period of 57 days, and no latitudinal motion. As for the latitudinal motion, the correlation coefficient is small, and the amplitude is well below the resolution of our images.

- Type II: This has a period of 86 days, and probably latitudinal motion. As for the latitudinal motion, the correlation efficient is small, but the peak-to-peak amplitude is almost at the limit of our resolution.

There seems to be a relation between the longitudinal and latitudinal motions for the 86-day component. That is, when the GRS increases in longitude (moves westward), it locates equatorward, where the zonal flow is westward. The GRS moves westward due to this zonal flow. When the GRS moves eastward, it locates poleward, where the zonal flow is eastward. The eastward motion of the GRS is due to this zonal flow. Hence, the relation of latitudinal and longitudinal motions of the GRS may be interpreted that the GRS is carried by the zonal flow around it.

\section{Numerical Experiments}

Numerical experiments were carried out using the equation of intermediate scale eddies in incompressible fluid (Yamagata, 1982; Williams and Yamagata, 1984; Asada, 1985b). We call this equation the IG (Intermediate Geostrophic) equation. If $\eta$ is the normalized surface elevation, $X$ is $x+t$ the eastward coordinate, $y$ is northward, $T$ is time with a long-time scale, the equation is

$$
\frac{\partial \eta}{\partial T}+\gamma J\left(\nabla^{2} \eta, \eta\right)-\frac{1}{\epsilon} \nabla^{2} \frac{\partial \eta}{\partial X}-\gamma \epsilon \eta \frac{\partial \eta}{\partial X}+2 y \frac{\partial \eta}{\partial X}=0
$$

where

$$
\nabla^{2}=\frac{\partial^{2}}{\partial X^{2}}+\frac{\partial^{2}}{\partial y^{2}} \quad \text { and } \quad J(A, B)=\frac{\partial A}{\partial X} \frac{\partial B}{\partial y}-\frac{\partial A}{\partial y} \frac{\partial B}{\partial X},
$$

and $\gamma=R_{o} / \delta^{2}, \epsilon=F \delta$ where $R_{o}$ is the Rossby number, $\delta$ is the beta parameter, and $F$ is the rotational Froude number. (For details, see Asada, 1985b.)

In our experiments, 65 grids were used in the longitudinal direction and 21 grids in the latitudinal direction. A rigid walls were assumed at the north and south boundaries, which was placed at $y= \pm 3.25$ times the eddy radius and may reflect waves. The cyclic condition was adopted at the east and west boundaries, and the bottom was assumed to be flat. We put an eddy of the Gaussian profile at the center of our calculation region. This eddy ejected a weak wave (inertia-gravity wave), and became stable.

In Fig. 6, we show the results of our experiments. These were calculated using the parameter for the Northern Hemisphere, so we need to think upside down to compare with the GRS. Zonal wind has a uniform shear, and the nondimensional velocity at the eddy is -1 . At this velocity, the vortex stretching through the $\beta$ effect around the eddy, is equal to the surface gradient of the zonal wind. Hence, the eddy is stable and has a long lifetime (Asada, 1985b).

In Fig. 7, we show the same results but have subtracted the zonal flow component from the results of Fig. 6. The inertia-gravity wave can be seen in this figure. From the figure, we calculated the position of the eddy as the weighted mean of position at which the surface elevation is more than $50 \%$ of the maximum value. The results are shown in Fig. 8. The upper figure is for the position and in the lower one we show the position from which is subtracted the constant speed component.

In this figure, the latitude of the eddy is seen to be high when it moves eastward, and low when it moves westward. This is consistent with the zonal wind profile, in which the 


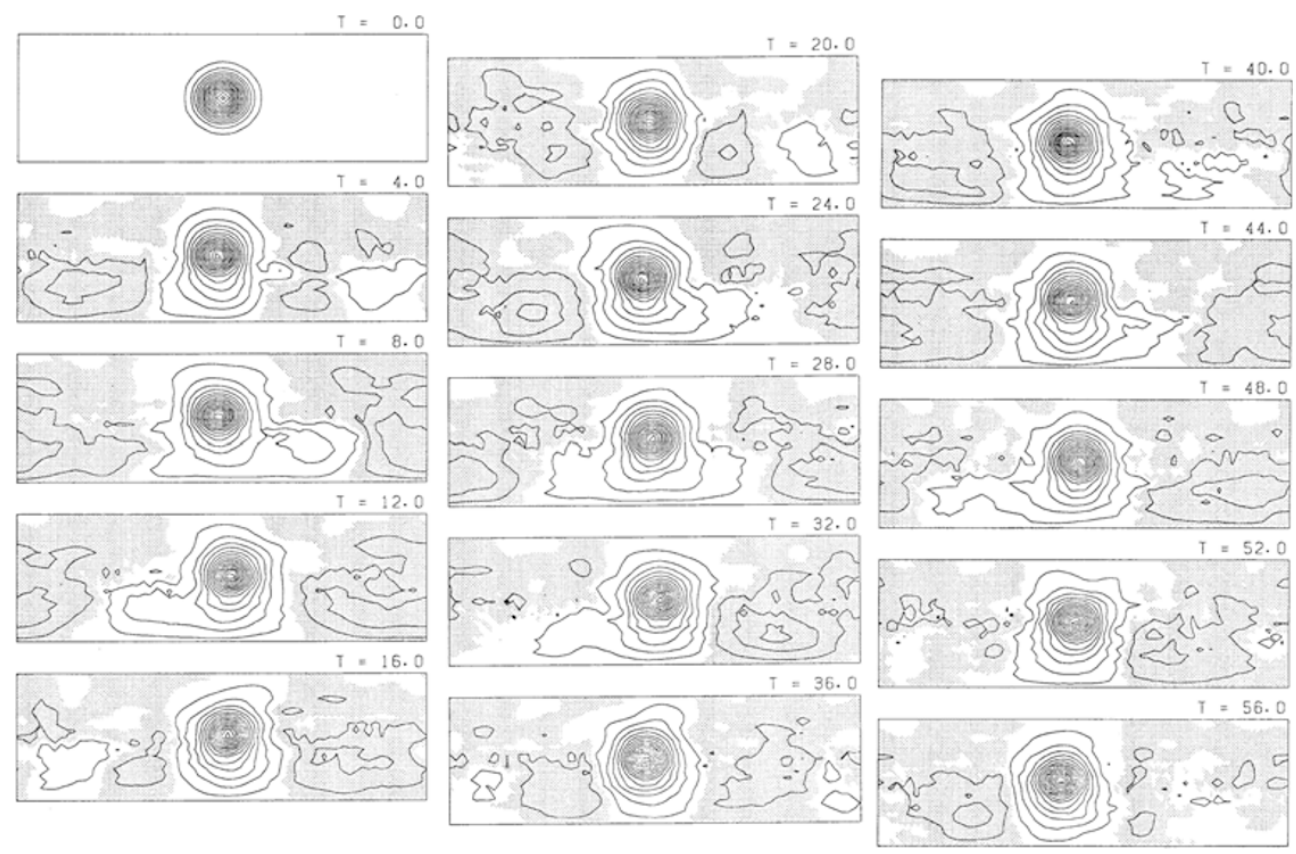

Fig. 7. The same as Fig. 6, but the zonal wind component was removed. Faint surface elevation due to inertia-gravity wave can be seen. The gray region has a negative value of elevation.

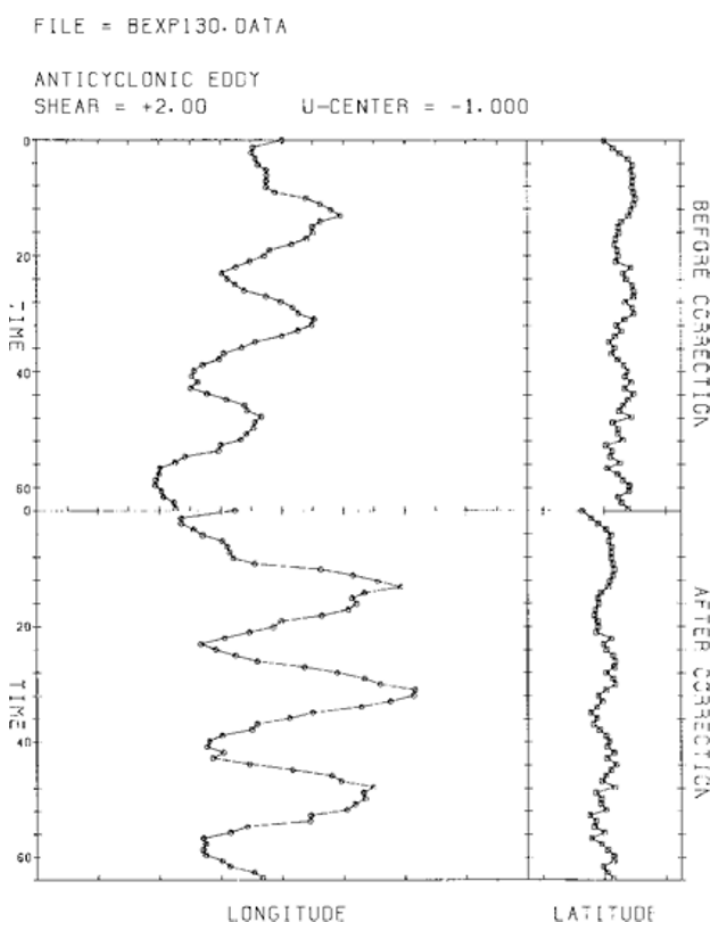

Fig. 8. Position of the eddy. In the upper figure, the position of the eddy is shown. In lower figure, we show the positions subtracted from the constant speed component. The scale interval of the horizontal axis corresponds to the grid interval of our calculation.

westward speed is slow in high latitude and fast in low latitude. Also this is the same as the observation of the 86day period described above.

Time variation of the surface elevation of the central latitude is shown in Fig. 9. Here, the weak wave is seen to collide with the eddy, when its motion changes from eastward to westward.

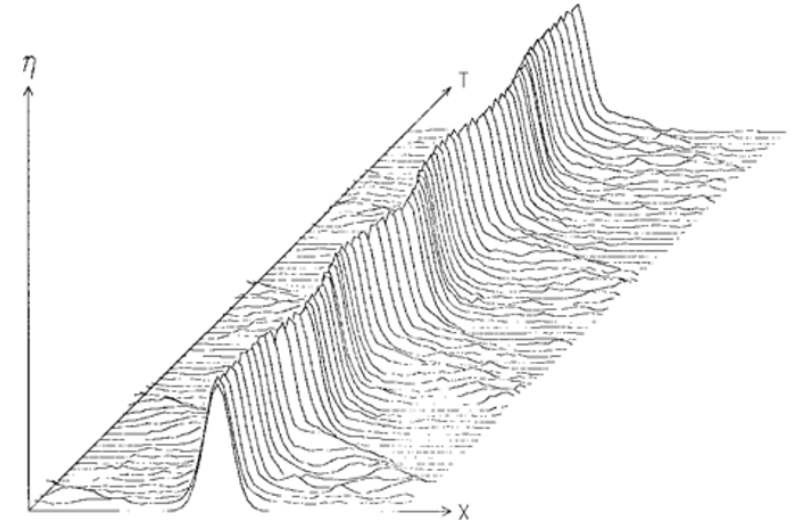

Fig. 9. Time variation of the surface profile at the central latitude. When the inertia-gravity wave collides with the eddy from right, the eddy can be seen to move left.

\section{Discussion}

The 57-day period component and the 86 days are suggested in the oscillating motion of the GRS of Jupiter. The 86-day component's behavior seems to correspond to our numerical experiments results.

Our observation results of 86 days and the numerical experiments suggest that when the GRS moves westward, the latitude of the GRS is relatively low, and that when it moves eastward, the latitude is high. Numerical experiments show that a wave collides with the GRS when the GRS changes its motion from eastward to westward.

We need to check the applicability of the IG equation to the GRS. The IG equation is said to be valid if the scale of phenomena, $L$, is

$$
L^{3}=L_{\beta} L_{d}^{2},
$$

where $L_{\beta}$ is the $\beta$ scale, $f_{0} / \beta$, is about $3.06 \times 10^{4} \mathrm{~km}$, if we adopt the Coriolis parameter, $f_{0}$, as $1.41 \times 10^{-4} \mathrm{sec}^{-1}$ and 
the gradient of the Coriolis parameter, $\beta$, as $4.61 \times 10^{-12}$ $\mathrm{sec}^{-1} \mathrm{~m}^{-1} . L_{d}$ is the Rossby deformation radius. If we use $L$ as $6000 \mathrm{~km}$, which is the latitudinal radius of the GRS, $L_{d}$ is calculated as $2.66 \times 10^{3} \mathrm{~km}$. In other words, if $L_{d}$ is around $2.66 \times 10^{3} \mathrm{~km}$, the IG equation is valid to the GRS. This value of $L_{d}$ is slightly large compared to the value from the inertia-gravity wave in the Shoemaker-Levy 9 (SL9) impact on Jupiter (Hammel et al., 1995). But it is much larger than the value that is estimated from the NTB activity (Asada et al., 1993). The deformation radius depends both on the static stability and the depth of the atmosphere, as $L_{d}=N H / f_{0}$, where $N$ and $H$ are the Brunt-Väisälä frequency and the vertical lengthscale. We think the GRS and SL9 event may be deep phenomena, whereas the NTB activity may be shallow phenomena. If we assumed that $N$ is constant, having a value of 0.01 which is used for the 0.6 bar level in the simulation by García-Melendo et al. (2005), we can estimate the vertical scale $H=38 \mathrm{~km}$ for the GRS using the above value of $f_{0}$ and $L_{d}$.

Another check is about zonal flow speed, in which the IG eddy has a long lifetime. The non-dimensional velocity of -1 corresponds to a dimensional velocity of $-\beta L_{d}^{2}$. If we adopt the $L_{d}$ value of $2.66 \times 10^{3} \mathrm{~km}$,

$$
-\beta L_{d}^{2}=-32.6 \mathrm{~m} \mathrm{sec}^{-1}
$$

which is greater than the peak velocity of the westward flow, $-50 \mathrm{~m} \mathrm{sec}^{-1}$. This means that at some latitude, which is the polar side of the westward peak jet of $20^{\circ} \mathrm{S}$, the zonal velocity is equal to $-\beta L_{d}^{2}$, where an IG eddy has a long lifetime.

If the results of our measurements and experiments are correct for the GRS, the oscillation of the GRS seems to be due to the collision of waves. At the latitude of the GRS, there is a westward jet of $-50 \mathrm{~m} / \mathrm{sec}$. If we divide the length of the latitude circle of the GRS by the above speed, it becomes 92.6 days. In other words, a particle in this westward jet collides with the GRS every 92.6 days, which is almost the same as the oscillation period.

Acknowledgments. We thank the two referees for their useful comments. This work is supported by the special personal research fund of Kyushu International University.

\section{References}

Asada, T., Longitudinal oscillation of the Jovian markings and numerical experiments of the intermediate scale eddies, Yahata University's Institute of Social Science and the Humanities Kiyo, 16, 195-228, 1985 (in Japanese).

Asada, T., Numerical experiments of the intermediate scale eddies and the STrD on Jupiter, J. Meteor. Soc. Japan, 63, 359-376, 1985.

Asada, T., P. J. Gierasch, and T. Yamagata, Initial development of eddies in hi-speed zonal flow: One interpretation for NTB activity of Jupiter, Icarus, 104, 60-68, 1993.

García-Melendo, E., et al., Jupiter's $24^{\circ} \mathrm{N}$ highest speed jet: Vertical structure deduced from nonlinear simulation of a large-amplitude natural disturbance, Icarus, 176, 272-282, 2005.

Hammel, H. B., et al., HST imaging of atmospheric phenomena created by the impact of comet Shoemaker-Levy 9, Science, 267, 1288-1296, 1995.

Reese, E. J., Jupiter's Red Spot in 1968-1969, Icarus, 12, 249-257, 1970.

Reese, E. J., Jupiter: Its Red Spot and other features in 1969-1970, Icarus, 14, 343-354, 1971

Reese, E. J., Jupiter: Its Red Spot and disturbances in 1970-1971, Icarus, 17, 57-72, 1972.

Reese, E. J. and R. F. Beebe, Measurements of Jupiter's long-lived features and currents, Contrib. Obs. of NMSU, 1, 176-191, 1976.

Solberg, H. G., A 3-month oscillation in the longitude of Jupiter's Red Spot, Planet. Space Sci., 17, 1573-1580. 1969.

Trigo-Rodriguez, J. M., et al., The 90-day oscillation of Jupiter's Great Red Spot revisited, Planet. Space Sci., 48, 331-339, 2000.

Williams, G. P. and T. Yamagata, Geostrophic regimes, intermediate solitary vortices and Jovian eddies, J. Atmos. Sci., 41, 453-478, 1984.

Yamagata, T., A class of solution missed by quasi-geostrophic approximation, J. Oceanogr. Soc. Japan, 38, 236-244, 1982.

T. Asada (e-mail: asada@kiu.ac.jp) and I. Miyazaki 